ARTICLE

\title{
Giant phonon anomalies in the proximate Kitaev quantum spin liquid $\alpha-\mathrm{RuCl}_{3}$
}

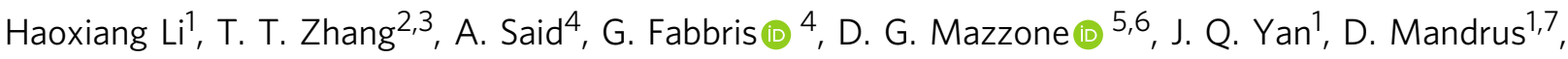

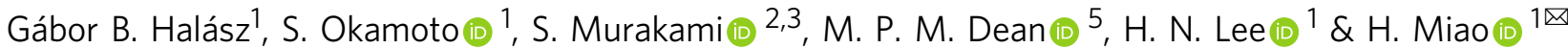

The Kitaev quantum spin liquid epitomizes an entangled topological state, for which two flavors of fractionalized low-energy excitations are predicted: the itinerant Majorana fermion and the $Z_{2}$ gauge flux. It was proposed recently that fingerprints of fractional excitations are encoded in the phonon spectra of Kitaev quantum spin liquids through a novel fractionalexcitation-phonon coupling. Here, we detect anomalous phonon effects in $\alpha-\mathrm{RuCl}_{3}$ using inelastic $\mathrm{X}$-ray scattering with $\mathrm{meV}$ resolution. At high temperature, we discover interlaced optical phonons intercepting a transverse acoustic phonon between 3 and $7 \mathrm{meV}$. Upon decreasing temperature, the optical phonons display a large intensity enhancement near the Kitaev energy, $J_{K} \sim 8 \mathrm{meV}$, that coincides with a giant acoustic phonon softening near the $Z_{2}$ gauge flux energy scale. These phonon anomalies signify the coupling of phonon and Kitaev magnetic excitations in $\alpha-\mathrm{RuCl}_{3}$ and demonstrates a proof-of-principle method to detect anomalous excitations in topological quantum materials.

\footnotetext{
${ }^{1}$ Materials Science and Technology Division, Oak Ridge National Laboratory, Oak Ridge, TN, USA. ${ }^{2}$ Department of Physics, Tokyo Institute of Technology, Okayama, Meguro-ku, Tokyo, Japan. ${ }^{3}$ Tokodai Institute for Element Strategy, Tokyo Institute of Technology, Nagatsuta, Midori-ku, Yokohama, Kanagawa, Japan. ${ }^{4}$ Advanced Photon Source, Argonne National Laboratory, Argonne, IL, USA. ${ }^{5}$ Condensed Matter Physics and Materials Science Department, Brookhaven National Laboratory, Upton, NY, USA. ${ }^{6}$ Laboratory for Neutron Scattering and Imaging, Paul Scherrer Institut, Villigen, Switzerland. ${ }^{7}$ Department

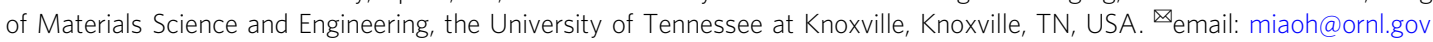


$\mathrm{n}$ correlated quantum materials, the nature of electronic interactions and their ground state topology is intimately linked to the geometry of the underlying lattice ${ }^{1-6}$. The lowenergy excitations arising from pure electronic degrees of freedom inevitably interact with the crystal lattice, leaving behind their fingerprints in the phonon spectrum. Hitherto, the interactions of phonons with "conventional" quasiparticles of either Bose-Einstein or Fermi-Dirac statistics, such as magnons in magnets $^{7}$, phasons and amplitudons in density waves ${ }^{8-10}$ and Bogoliubons in superconductors ${ }^{11}$, have been explored extensively. In contrast, the coupling between phonons and fractional excitations, including spinons in one-dimensional magnets ${ }^{1-3,12-15}$, and Majorana fermions (MFs) and $Z_{2}$ gauge fluxes that are thought to exist in the Kitaev quantum spin liquids (QSL) ${ }^{16-25}$, have remained elusive. The discovery of such fractional-excitation-phonon coupling (FPC) is of fundamental importance, as they carry key information of the intertwined quantum state ${ }^{12-15}$. In particular, the coupling of phonons to the itinerant MFs has been predicted to play a pivotal role in the realization of the field-induced quantum thermal Hall effect in $\alpha-\mathrm{RuCl}_{3}{ }^{26-29}$, which is a signature of quantum entanglement in Kitaev-QSLs ${ }^{30-32}$.

Numerous studies have shown that the low-temperature phase of $\mathrm{a}-\mathrm{RuCl}_{3}$ is a promising Kitaev-QSL candidate ${ }^{17-24,30-32}$. As displayed in Fig. 1a, the edge-sharing $\mathrm{Ru}-\mathrm{Cl}$ octahedra form an effective spin-1/2 honeycomb network. The destructive quantuminterference through the close-to- $90^{\circ} \mathrm{Ru}-\mathrm{Cl}-\mathrm{Ru}$ bonds significantly suppresses the Heisenberg magnetic exchange interaction, yielding a dominant Ising-type interaction $(\mathbf{J})$ perpendicular to the $\mathrm{Ru}-\mathrm{Cl}-\mathrm{Ru}$ plane $\mathrm{e}^{33}$ (Fig. 1b). Figure 1c schematically depicts the phase diagram of $\alpha-\mathrm{RuCl}_{3}$. At zero magnetic field, the lowenergy excitations in the paramagnetic phase are primarily determined by the Kitaev term ${ }^{3,4,17-24,30-32}$

$$
H=\sum_{\gamma,<i, j>} J_{K}^{\gamma} S_{i}^{\gamma} S_{j}^{\gamma}
$$

Here $J_{K}^{\gamma}(\gamma=X, Y, Z)$ is the bond-dependent coupling parameter, and $<i, j>$ stands for nearest-neighbor pairs of spins at one of the $X, Y$, or $Z$ bonds. The two characteristic energy scales are shown in Fig. 1d for the isotropic limit $\left(J_{K}^{\gamma}=J_{K}\right)$. Below the Kitaev temperature scale $T_{k} \sim J_{K}$, the low-energy excitations of Eq. (1) start to fractionalize into itinerant MFs and fluctuating $\mathrm{Z}_{2}$ gauge fluxes ${ }^{34}$. The former features a continuum that peaks broadly near $J_{\mathrm{K}}$, while the latter is a local excitation with an energy around $0.065 J_{\mathrm{K}}^{16,20,25,34}$. Below $T_{\mathrm{N}}=7 \mathrm{~K}$, non-Kitaev interactions such as remnant Heisenberg magnetic exchange couplings, stabilize zigzag antiferromagnetic order that is suppressed under magnetic field ${ }^{35-39}$. Above $\mathbf{B} \sim 7 \mathrm{~T}$, a quantized thermal Hall conductivity (red region in Fig.1c) is observed, indicating strongly an entangled topological phase $\mathrm{e}^{30-32}$. However, unlike the quantum Hall effect of electrons, it has been theoretically predicted that the quantum thermal Hall effect can only be approximate and requires strong $\mathrm{FPCs}^{26-29}$. Here we report experimental signature of the FPC in $\alpha-\mathrm{RuCl}_{3}$ by uncovering two-types of phonon anomalies at zero magnetic field: a $35 \%$ enhancement of the phonon spectral weight near the Kitaev energy $J_{K}$, and a giant phonon softening of $\sim 15 \%$ below $2 \mathrm{meV}^{26-29}$. a
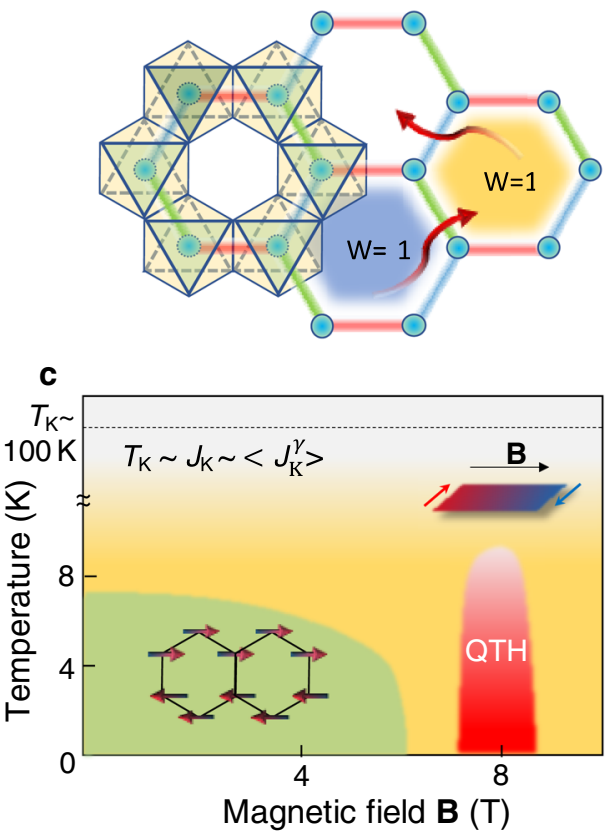

b

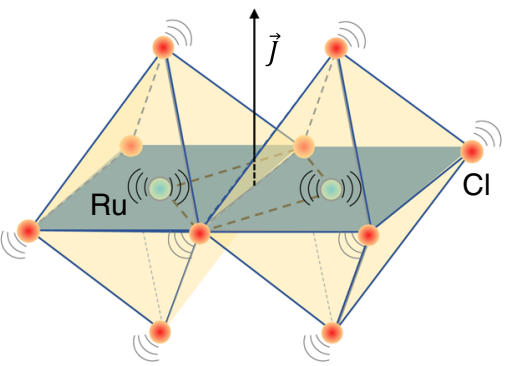

d

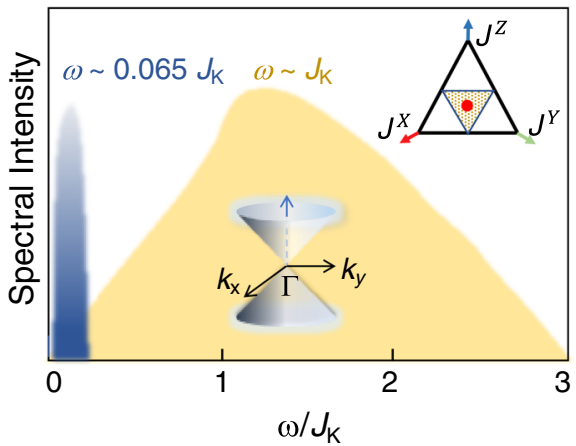

Fig. 1 Schematics of a Kitaev-QSL and the phase diagram of $\boldsymbol{\alpha}-\mathbf{R u C l}_{\mathbf{3}}$ a Structure motif of $\alpha-\mathrm{RuCl}_{3}$ based on a honeycomb lattice of edge-sharing Ru-Cl octahedra. The red, green, and blue bonds represent three orthogonal Kitaev interactions $J_{K}^{\gamma}$. In the pure Kitaev model [Eq. (1)], the low-energy excitations fractionalize into itinerant MFs (red arrows) and anyonic $Z_{2}$ flux (blue and yellow hexagons) $W= \pm 1$ represents the $Z_{2}$ index. $\mathbf{b}$ The nearly $90^{\circ} \mathrm{Ru}-\mathrm{Cl}-\mathrm{Ru}$ bonds and the moderate spin-orbit-coupling favor an Ising-type magnetic interaction that is perpendicular to the Ru-Cl-Ru plane highlighted in a blue-green color. Lattice vibrations perturbatively modify the magnetic interactions, which induce a coupling between phonons and fractional excitations. c illustrates the phase diagram of $\alpha-\mathrm{RuCl}_{3}$ on a logarithmic-scale: below $T_{\mathrm{K}} \sim J_{\mathrm{K}} \sim 8 \mathrm{meV}$ (the yellow area) the thermal Hall conductivity, $\kappa_{\mathrm{xy}}$, becomes finite, indicating a proximate Kitaev-QSL with $M F$ and $Z_{2}$ gauge flux. In the green area $\left(T<T_{N}=7 \mathrm{~K}\right)$, non-Kitaev terms drive the system into zigzag antiferromagnetic order. Under an external magnetic field $(\mathbf{B}>7 \mathrm{~T})$ that completely suppresses the magnetic order, the system is driven into a quantum thermal Hall state at finite temperature (the red area). $\mathbf{d}$ schematically shows two characteristic Kitaev energy scales in the isotropic limit: the itinerant MF excitation ${ }^{18}$ (yellow area) that is broadly peaked around $J_{K}$ and the $Z_{2}$ gauge flux excitations (blue area) near $0.065 J_{K}$. 

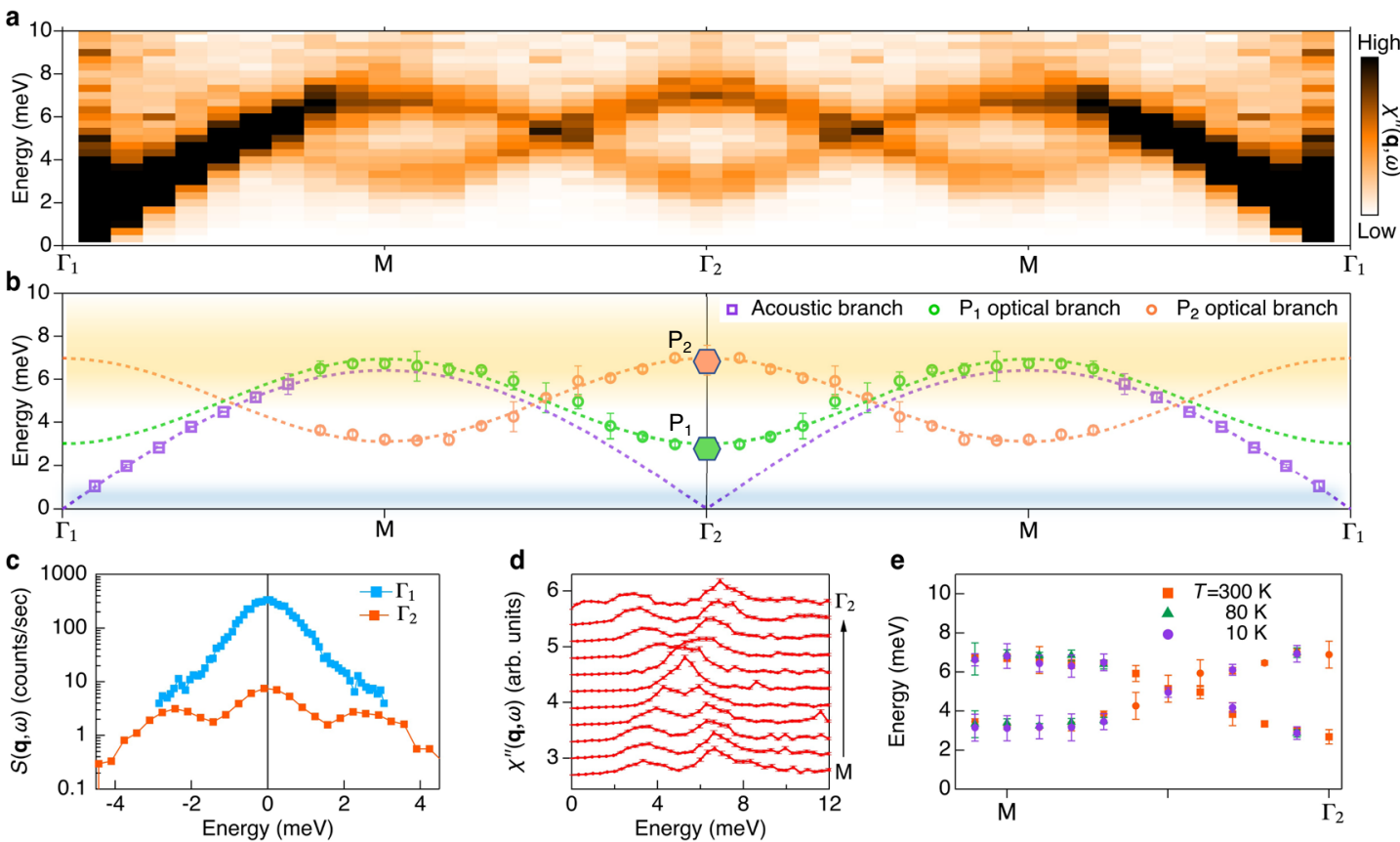

$\Gamma$

$\Gamma_{1}$
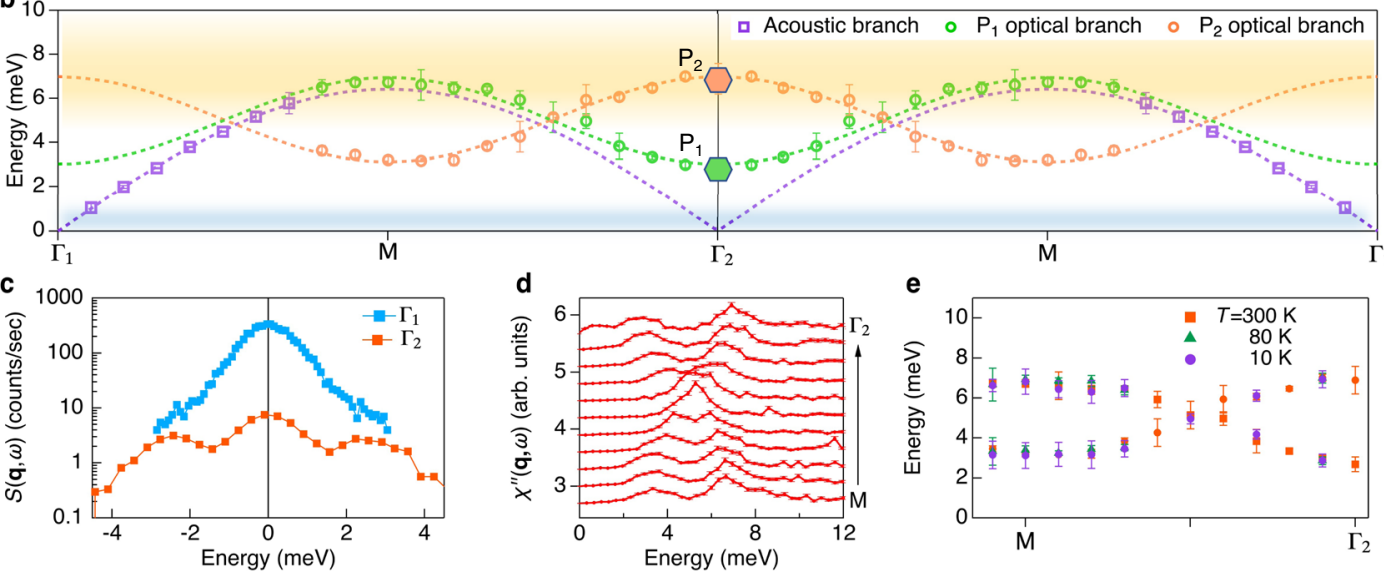

Fig. 2 Room temperature phonon excitations in $\boldsymbol{\alpha}-\mathbf{R u C l}_{\mathbf{3}}$. a Low-energy phonon excitations determined by IXS along the $\Gamma_{1}(6,-3,0)-M(6,-2.5,0)-\Gamma_{2}$ $(6,-2,0)$ direction. The plot shows the Bose-factor corrected IXS intensity. The extracted peak positions are presented in (b) revealing interlaced optical phonons intercepting with the transverse acoustic phonon branch. The optical phonon energies at $\Gamma_{2}$ are denoted by the green $\left(P_{1}\right)$ and orange $\left(P_{2}\right)$ hexagons, which are consistent with the phonon modes previously found by $\mathrm{THz}$-spectroscopy ${ }^{38}$. The yellow and blue shaded areas correspond to the two characteristic Kitaev energy scales displayed in Fig. 1d. c IXS spectra at $\Gamma_{1}$ and $\Gamma_{2}$. The intensity is shown on a logarithmic scale. Note that due to the large intensity difference at $\Gamma_{1}$ and $\Gamma_{2}$, the acoustic phonon intensity is expected to be extremely weak near $\Gamma_{2}$. $\mathbf{d}$ Constant momentum transfer cuts around the phonon-crossing. The two optical branches cross each other without imposing a hybridization gap. $\mathbf{e}$ The extracted phonon peak positions from $\mathrm{M}$ to $\Gamma_{2}$ at different temperatures reveal a temperature-independent massless Dirac-cone. The error bars in $\mathbf{b}$, e denote the $2 \sigma$ returned from the fittings (see Supplementary Note 2). The error bars in $\mathbf{d}$ represent one standard deviation assuming Poisson counting statistics.

\section{Results}

Figure 2a shows the imaginary part of the dynamical phonon susceptibility $\chi^{\prime \prime}(\mathbf{Q}, \omega)$ along $\Gamma_{1}(6,-3,0)-\mathrm{M}(6,-2.5,0)-\Gamma_{2}(6,-2,0)$ in reciprocal lattice units (r.l.u.) at room temperature (see Supplementary Note 6 for first-principles calculations of phonon dispersion). The dynamical susceptibility is given by the fluctuationdissipation theorem via $\chi^{\prime \prime}(\mathbf{Q}, \omega)=S(\mathbf{Q}, \omega) \times\left(1-e^{-\omega / k_{B} T}\right)$, where $S(\mathbf{Q}, \omega)$ is the dynamical phonon structure factor that is directly measured by inelastic $\mathrm{x}$-ray scattering (IXS). The total momentum transfer $\mathbf{Q}=\mathbf{q}+\mathbf{G}$, is composed of the reduced momentum transfer in the first Brillouin zone $\mathbf{q}$ and the reciprocal lattice vector $\mathbf{G}$. The elastic contribution at $\omega=0$ was subtracted by fitting the IXS raw data in the entire energy window (see Supplementary Note 1 and Note 2). We selectively probe in-plane transverse phonon modes, whose dispersions (open circles and open squares) and sinusoidal fits (dashed curves) are shown in Fig. 2b. As shown in Fig. 2a, the intensity of the transverse acoustic phonon changes significantly from $\Gamma_{1}$ to $\Gamma_{2}$, reflecting their different Bragg peak intensities that are plotted in Fig. 2c. Two low-energy optical phonons, $\mathrm{P}_{1}$ and $\mathrm{P}_{2}$, are observed at the Brillouin zone center $\Gamma_{2}$, corresponding to $\omega_{1}=2.7$ and $\omega_{2}=7 \mathrm{meV}$, which are in good agreement with previous optical and neutron studies ${ }^{19,40}$. The two optical phonons carry opposite phonon velocities and form an interlaced structure that intercepts the acoustic phonon. An apparent phonon crossing occurs between $\Gamma$ and $\mathrm{M}$ (Fig. 2d and e), suggesting possible Dirac-cone and topological phononic nodal-lines ${ }^{41,42}$.

In $a-\mathrm{RuCl}_{3}, J_{\mathrm{K}}$ is estimated to be $5-9 \mathrm{meV}$ in the lowtemperature phase below $150 \mathrm{~K}^{4,17-25,30}$ (more discussions in Supplementary Note 5), which roughly corresponds to the top of the $\mathrm{P}_{1}-\mathrm{P}_{2}$ phonon band. Thus, if Majorana-phonon coupling is present, phonon anomalies are expected in the energy range shown in Fig. 2b. Moreover, a recent theoretical study of the pure Kitaev model predicts that the Majorana-phonon coupling is momentum dependent and peaks near the $\mathrm{M}$ and $\mathrm{K}$ point ${ }^{28}$. To uncover the energy and momentum-dependent coupling between the optical phonons and the suggested MFs, we compare the temperature-dependent $\chi^{\prime \prime}(\mathbf{q}, \omega)$ along the $\mathrm{M}-\Gamma_{2}$ path. A large spectral enhancement can be observed clearly in Fig. 3a-f. Near the $\mathrm{M}$ point, the peak intensity of $\mathrm{P}_{1}$ increases dramatically upon cooling from $300 \mathrm{~K}$ to $10 \mathrm{~K}$. In contrast, the peak intensity of $P_{2}$ is unchanged except the $10 \mathrm{~K}$ data at the $\mathrm{M}$ point. When approaching the $\Gamma_{2}$ point (towards larger $|\mathbf{q}|$ ), the intensity enhancement first decreases near the crossing-point $\left(\mathrm{P}_{1}\right.$ and $\mathrm{P}_{2}$ crossed at $q=0.75)$, but then reappears at $\mathrm{P}_{2}$, which is higher in energy near the $\Gamma_{2}$ point. Interestingly, we find that the spectral enhancement is different between the symmetry related points $q=0.45$ and $q=0.55$. As we show in Fig. $2 \mathrm{a}$, the transverse acoustic phonon starts to merge with the optical phonon near the $\mathrm{M}$ point. Since the acoustic phonon intensity is stronger at $q=0.45$, the asymmetric intensity enhancement suggests that the Majorana-phonon coupling is larger on the acoustic mode than the optical mode near $\omega \sim J_{K}$. To quantitatively show the spectral enhancement effect, we extract the temperatureinduced difference in the integrated phonon intensity, $\Delta \chi^{\prime \prime}\left(\mathbf{q}, \omega_{0}\right)=\int_{\omega_{0}-\infty}^{\omega_{0}+\infty}\left[\chi^{\prime \prime}(\mathbf{q}, \omega, 10 \mathrm{~K})-\chi^{\prime \prime}(\mathbf{q}, \omega, 300 \mathrm{~K})\right] d \omega$, and plot $\Delta \chi^{\prime \prime}\left(\mathbf{q}, \omega_{0}\right)$ as function of $\Delta E=\omega_{0}-\omega_{\max }$ in Fig. 3g. Here $\omega_{0}$ denotes the phonon peak position and $\omega_{\max }=7 \mathrm{meV}$ is the band-top energy of $\mathrm{P}_{1}$ and $\mathrm{P}_{2}$. Unlike the broad continuum observed in the spin correlation function ${ }^{17-19,21-23}, \Delta \chi\left(\mathbf{q}, \omega_{0}\right)$ decreases rapidly as $\omega_{0}$ moves away from $J_{\mathrm{K}}$ (Fig. 3). It also shows strong momentum dependence with the enhancement occuring around the high symmetry points $\mathrm{M}$ and $\Gamma_{2}$ (see Supplementary 

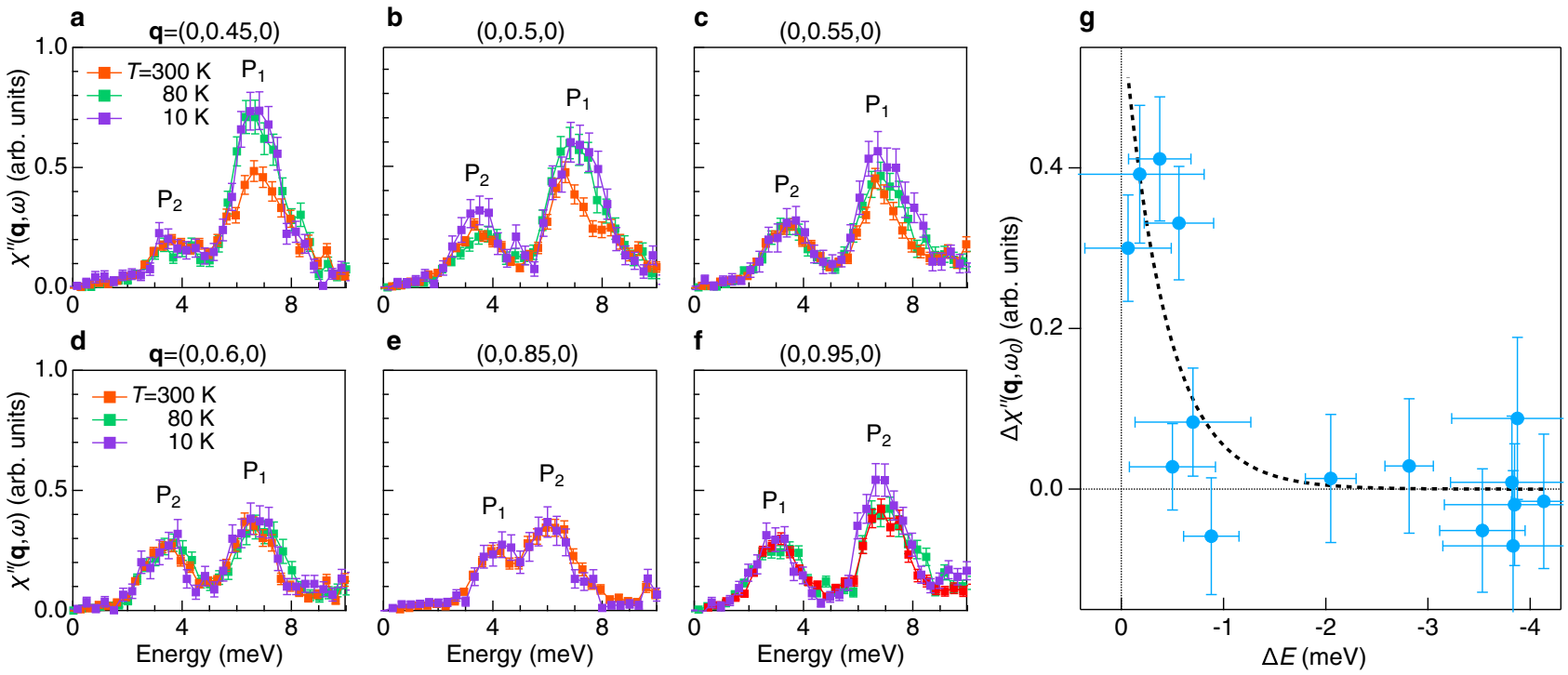

Fig. 3 Itinerant MF-phonon coupling near $\boldsymbol{\omega} \sim \boldsymbol{J}_{\mathbf{K}}$. a-f Spectra of the interlaced optical phonons at different reduced momentum transfer $\mathbf{q}$. Here, we define $\mathbf{q}=(0,0,0)$ and $(0,1,0)$ as $\Gamma_{1}$ and $\Gamma_{2}$, respectively, where the $M$ point is at $\mathbf{q}=(0,0.5,0)$. The labels $P_{1}$ and $P_{2}$ denote the two optical phonon branches. Note the relative peak position of $P_{1}$ and $P_{2}$ switches at $\mathbf{q}=(0,0.75,0)$. The temperature dependent $\chi^{\prime \prime}(\mathbf{q}, \omega)$ shows a spectral weight enhancement at $\omega \sim J_{K}$ at low temperature. In $\mathbf{b}$ we notice a shoulder on $P_{2}$ that may come from the acoustic mode. $\mathbf{g}$ The difference in the integrated phonon spectral weight, $\Delta \chi \prime \prime\left(\mathbf{q}, \omega_{0}\right)$, between 10 and $300 \mathrm{~K}$ as a function of $\Delta E=\omega_{0}-\omega_{\max }$. Here $\omega_{0}$ is the phonon peak position, $\omega_{\max }=7 \mathrm{meV}$ is the band top of the interlaced optical phonons. The drastic increase of $\Delta \chi^{\prime \prime}\left(\mathbf{q}, \omega_{0}\right)$ is fitted to an exponential function (dashed line). The vertical error bars in all panels represent one standard deviation based on Poisson counting statistics. The horizontal error bars in $\mathbf{g}$ denote the $2 \sigma$ returned from the fitting algorithm that extract the spectral peak positions.

Fig. 9). This observation is in qualitative agreement with theoretical calculation that shows energy and momentum dependent Majorana-phonon coupling ${ }^{28}$ (spectrum near the $\mathrm{K}$ point with spectral peak at higher energy is shown in Supplementary Fig. 6). The observed phonon enhancement is also consistent with a recent study of frustrated magnetic systems, which predicts large IXS cross-section for magnetic excitations ${ }^{7}$. We note, however, a quantitative understanding of the energy and momentumdependent optical phonon enhancement may require theoretical calculations beyond the pure Kitaev model.

We then turn to the transverse acoustic phonon near $\Gamma_{1}$. Figure $4 \mathrm{a}$ and $\mathrm{b}$ show the temperature-dependence of $\chi^{\prime \prime}(\mathbf{q}, \omega)$ at $\mathbf{q}_{\mathbf{1}}=(0,0.1,0)$ (or $\left.\mathbf{Q}_{\mathbf{1}}=(6,-2.9,0)\right)$ and $\mathbf{q}_{2}=(0,0.15,0)$ (or $\mathbf{Q}_{2}=(6,-2.85,0)$ ), respectively. At $\mathbf{q}_{1}$, the phonon peak position gradually shifts to lower energies. In contrast, it remains nearly unchanged at $\mathbf{q}_{2}$. The softening-effect is confirmed by directly comparing the raw data, $S(\mathbf{q}, \omega)$, at 10 and $300 \mathrm{~K}$ (Fig. $4 \mathrm{c}$ and d). The peak position is softened by about $13 \%$ at $\mathbf{q}_{\mathbf{1}}$, which corresponds to $\sim 0.3 \mathrm{meV}$ shift in energy. Figures $4 \mathrm{e}$ and $\mathrm{f}$ show the relative peak shift $\omega_{0}(T) / \omega_{0}(300 \mathrm{~K})$ at $\mathbf{q}_{1}$ and $\mathbf{q}_{2}$ as function of temperature. We find that the acoustic phonon softening at $\mathbf{q}_{\mathbf{1}}$ becomes progressively stronger below $80 \mathrm{~K}$, consistent with the thermal Hall effect in $\alpha-\mathrm{RuCl}_{3}$ where the thermal Hall conductivity, $\kappa_{\mathrm{xy}}$, starts to increase. In Fig. $4 \mathrm{e}$, we further show the phonon softening at $\mathbf{q}_{3}=(0,0.05,0)$. The error-bars returned from fittings are larger at $\mathbf{q}_{3}$ as the elastic intensity becomes stronger when approaching the Bragg peak. Interestingly, the relative phonon softening at $\mathbf{q}_{\mathbf{3}}(\sim 15 \%)$ is even larger when compared to $\mathbf{q}_{\mathbf{1}}$. This suggests an enhanced renormalization for long wavelength acoustic phonons.

\section{Discusson}

The discovery of temperature and energy dependent phonon softening provides important information on the FPC in $\alpha-\mathrm{RuCl}_{3}$.
In the pure Kitaev model [Eq. (1)], quantum fractionalization occurs at $T_{\mathrm{K}} \sim J_{\mathrm{K}} \sim 100 \mathrm{~K}^{43}$, in agreement with our observations. Below $T_{K}$, the dispersionless gauge flux excitation crosses the linear dispersing acoustic phonon near $\omega=0.065 J_{K} \sim 0.5 \mathrm{meV}^{23}$ and induces a phonon anomaly near this energy scale (Fig. $4 \mathrm{~g}$ ). The observation of enhanced phonon softening $\left[\omega\left(\mathbf{q}_{1}\right)=2 \mathrm{meV}\right.$ and $\omega\left(\mathbf{q}_{3}\right)=1 \mathrm{meV}$ as $\omega \rightarrow 0.065 J_{K}$ is consistent with this picture, where the softening effect is expected to be significantly suppressed for $\omega(\mathbf{q}) \gg 0.065 J_{K}$. Figure $4 \mathrm{~h}$ depicts another scenario that attempts to explain the phonon-softening. Here, the acoustic phonon and the itinerant MFs possess nearly identical linear dispersions at $\mathbf{q} \rightarrow 0^{29}$. This enhances Majorana-phonon coupling that yields a renormalization of the phonon dispersion below $T_{\mathrm{K}}{ }^{28,29}$. To justify this conjecture, we extract the acoustic phonon velocity $v_{\mathrm{ph}} \sim 16 \mathrm{meV} \AA(\hbar=1)$, which is based on the room-temperature phonon dispersion shown in Fig. 2. In the isotropic limit ${ }^{16}$, the velocity of the itinerant MF is $v_{\mathrm{MF}}=\frac{\sqrt{3}}{4} J_{K} a$, where the in-plane lattice constant $a=5.9639 \AA$. Comparing $v_{\mathrm{ph}}$ and $v_{\mathrm{MF}}$ gives $J_{K} \sim 6.2 \mathrm{meV}$, comparable to the experimental value. Besides the $Z_{2}$ gauge flux and MFs, in more realistic models with non-Kitaev interactions ${ }^{44,45}$, other fractional excitations may also be consistent with the observed phonon anomalies. It is important to note that the charge and magnetic excitations below $2 \mathrm{meV}$ still remain unresolved in $\mathrm{a}-\mathrm{RuCl}_{3}$. In particular, direct experimental evidence of $Z_{2}$ gauge flux is not well established yet. The observed acoustic phonon softening below $2 \mathrm{meV}$ demonstrate a small energy scale in $\alpha-\mathrm{RuCl}_{3}$ that strongly renormalizes the acoustic phonon spectrum and hence may be responsible for the quantized thermal Hall effect.

Finally, we discuss the possibility of magnon-phonon coupling. Below $T_{\mathrm{N}}$, a gapped magnon excitation between $2-7 \mathrm{meV}$ was observed in $\mathrm{a}-\mathrm{RuCl}_{3}$ by previous neutron studies ${ }^{19,21,22,37}$. However, as we show in Figs. 3 and 4, the phonon anomalies onset at $T_{\mathrm{K}}$, which is well above $T_{\mathrm{N}}$. More importantly, evidence of an enhanced 

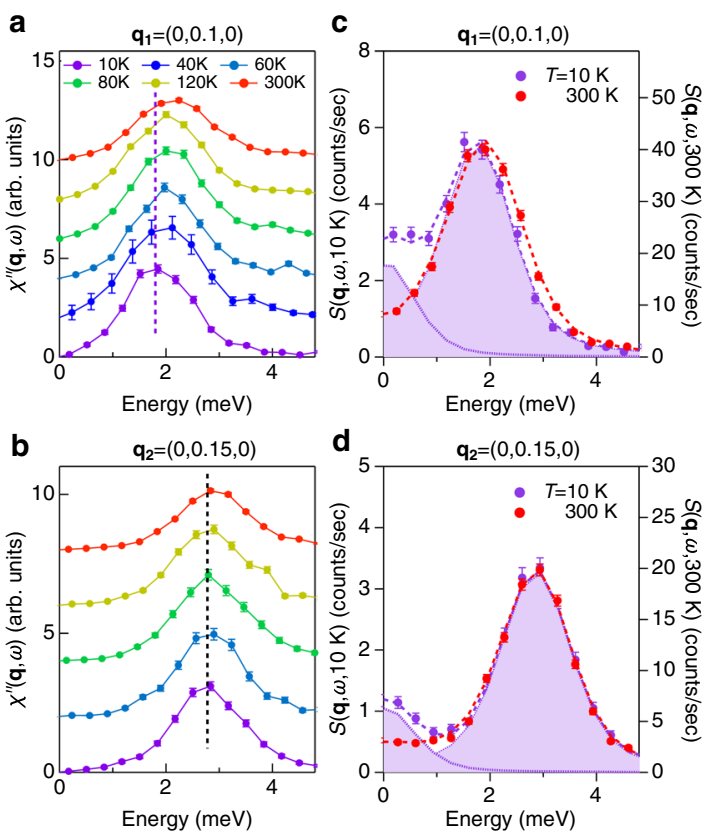

e

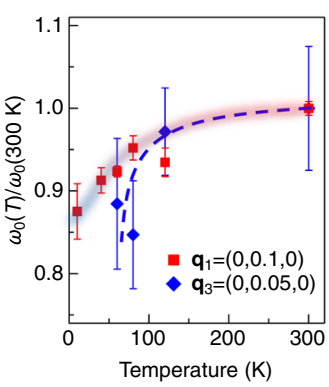

f

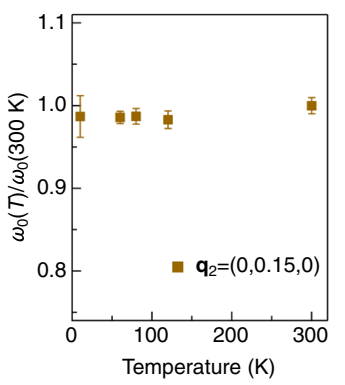

g

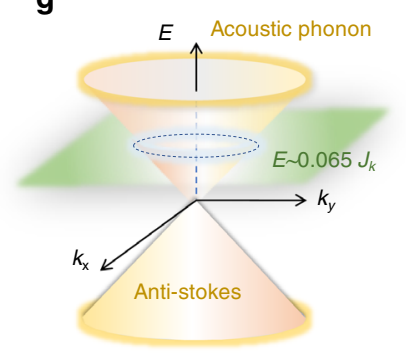

h

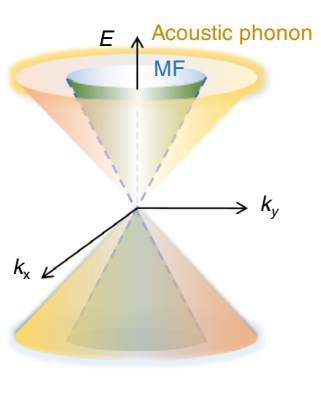

Fig. 4 Giant acoustic phonon softening. a, b Show the temperature-dependent $\chi^{\prime \prime}(\mathbf{q}, \omega)$ at $\mathbf{q}_{\mathbf{1}}=(0,0.1,0)$ in reciprocal lattice units (r.l.u.), $\omega \sim 2$ meV and $\mathbf{q}_{\mathbf{2}}=(0,0.15,0)$ r.l.u., $\omega \sim 3 \mathrm{meV}$, respectively. $\mathbf{c}$ direct comparison of the IXS raw data, $S\left(\mathbf{q}_{\mathbf{1}}, \omega\right)$, at $T=10$ and $300 \mathrm{~K}$. $\mathbf{d}$ shows the same plot as $(\mathbf{c})$ but at $\mathbf{q}_{\mathbf{2}}$. There is an apparent phonon softening at $\mathbf{q}_{\mathbf{1}}$, while at $\mathbf{q}_{\mathbf{2}}$, the effect is negligible. $\mathbf{e}, \mathbf{f}$ The relative peak shift at $\mathbf{q}_{\mathbf{1}}, \mathbf{q}_{\mathbf{2}}$ and $\mathbf{q}_{\mathbf{3}}$. The $\sim 13 \%$ phonon softening at $\mathbf{q}_{\mathbf{1}}$ (red squares in $\mathbf{e}$ ) corresponds to a $\sim 0.3 \mathrm{meV}$ phonon peak shift. This value is as large as some well-known electron-phonon coupled systems ${ }^{10}$. The blue diamonds in e represent the relative peak shifts at $\mathbf{q}_{\mathbf{3}}=(0,0.05,0)$ that show even larger softening-effect $(\sim 15 \%$ at $60 \mathrm{~K})$, whereas $\mathbf{q}_{\mathbf{2}}$ displays negligible change as shown in (f). This acoustic phonon anomaly, together with the spectral enhancement discussed in Fig. 3, present a full picture of the FPC in $\alpha-$ $\mathrm{RuCl}_{3}$. $\mathbf{g}$, h Schematically show two phonon coupling mechanisms. $\mathbf{g}$ The flatband of the $Z_{2}$ flux mode intercepts the acoustic phonon near $\omega \sim 0.065 J_{K}$. $\mathbf{h}$ The nearly identical linear dispersion of the itinerant MF and the acoustic phonon at $\mathbf{q} \rightarrow 0$ causes a phonon renormalization at low temperature. The error bars in a-d represent one standard deviation assuming Poisson counting statistics. The error bars in $\mathbf{e}, \mathbf{f}$ denote the $2 \sigma$ returned from the fitting.

phonon softening is observed at $\omega=1 \mathrm{meV}$ (see $\mathbf{q}_{3}$ in Fig. $4 \mathrm{e}$ and Supplementary Fig. 5), which is well below the magnon gap. Therefore, a magnon-phonon coupling is unlikely giving rise to the observed acoustic phonon softening. However, the magnon-phonon coupling may indeed be present in $\alpha-\mathrm{RuCl}_{3}$. As we show in Fig. 2, the $\mathrm{P}_{2}$ phonon energy is the same as the magnon energy near the $M$ point ${ }^{19,21,38}$. Interestingly, the $P_{2}$ phonon intensity at the $M$ point is enhanced at $10 \mathrm{~K} \sim T_{\mathrm{N}}$, supporting magnon-phonon coupling ${ }^{46}$. In addition, strong anharmonicity is proposed in the magnon excitation of this material ${ }^{47}$, which represents the break-down of the spin quasiparticles. Such excitations contain extremely broad features ${ }^{47}$ that are contradictory to the well-defined energy scale of the phonon anomalies observed here.

Our discovery of two-types of phonon anomalies, i.e., the spectral enhancement in the optical phonon and the acoustic mode softening, provides experimental signature of FPC in the proximity of Kitaev-QSL ${ }^{26,27}$. Beyond the aforementioned implications, our observation has an even deeper impact on correlated topological quantum states. First of all, our approach can be immediately applied to other Kitaev-QSL candidates ${ }^{1,3,4}$, such as iridates ${ }^{4,48}$, where the inelastic neutron scattering experiments are difficult to perform due to strong neutron absorption of Ir. Moreover, it has been predicted that in $U(1)$ spin liquids the spinon Fermi surface features a large singularity at $2 \mathbf{k}_{\mathrm{F}}$, which induces phonon anomalies at $\mathbf{q}=2 \mathbf{k}_{\mathrm{F}}^{12}$. Both kagome and triangular lattices have been speculated to host such charge neutral Fermi surfaces ${ }^{49,50}$. More recently, a giant thermal Hall effect has been observed in the cuprate high- $T_{\mathrm{c}}$ superconductors $^{13}$ with large phonon contributions ${ }^{51}$. While mechanisms based on chiral spin liquid or topological spinons ${ }^{14,15}$ have also been proposed, the theoretically predicted $\kappa_{\mathrm{xy}}$ is $50 \%$ smaller than the experimental value ${ }^{14}$, suggesting large phonon effect. Our observation of FPC in $\alpha-\mathrm{RuCl}_{3}$ validates phonons as a sensitive probe to uncover hidden fractional and non-local excitations, and hence can help resolving key puzzles in correlated and entangled quantum states.

\section{Methods}

Sample preparation and characterizations. Millimeter-sized $\alpha-\mathrm{RuCl}_{3}$ crystals were grown by the sublimation of $\mathrm{RuCl}_{3}$ powder sealed in a quartz tube under vacuum ${ }^{52}$. The growth was performed in a box furnace. After dwelling at $1060^{\circ} \mathrm{C}$ for $6 \mathrm{~h}$, the furnace was cooled to $800^{\circ} \mathrm{C}$ at $4{ }^{\circ} \mathrm{C} / \mathrm{h}$. Magnetic order was confirmed to occur at $7 \mathrm{~K}$ by measuring magnetic properties and specific heat ${ }^{21}$.

Inelastic X-ray scattering. The experiments were conducted at beam line 30-ID-C (HERIX) at the Advanced Photon Source (APS). The highly monochromatic X-ray beam of incident energy $E_{\mathrm{i}}=23.7 \mathrm{keV}(\mathrm{l}=0.5226 \AA)$ was focused on the sample with a beam cross section of $\sim 35 \times 15 \mathrm{~mm}^{2}$ (horizontal $\times$ vertical). The total energy resolution of the monochromatic X-ray beam and analyzer crystals was $\Delta \mathrm{E} \sim 1.3$ $\mathrm{meV}$ (full width at half maximum). The measurements were performed in transmission geometry. Typical counting times were in the range of 30-120 s per point in the energy scans at constant momentum transfer $\mathbf{Q}$. H, K, L are defined in the trigonal structure with $\mathrm{a}=\mathrm{b}=5.9639 \AA, \mathrm{c}=17.17 \AA$ at the room temperature.

Density functional theory calculations of phonon spectrum. Phonon dispersions for $\alpha-\mathrm{RuCl}_{3}$ were calculated using with density functional perturbation theory (DFPT) and the Vienna Ab initio Simulation Package (VASP). The exchangecorrelation potential was treated within the generalized gradient approximation (GGA) of the Perdew-Burke-Ernzerhof variety, where the kinetic energy cutoff was set to $400 \mathrm{eV}$. Integration for the Brillouin zone was done by using a MonkhorstPack $k$-point grids which is equivalent to $8 \times 8 \times 9$.

\section{Data availability}

The data that support the findings of this study are available from the corresponding author on reasonable request. 
Received: 5 April 2021; Accepted: 30 April 2021;

Published online: 10 June 2021

\section{References}

1. Balents, L. Spin liquids in frustrated magnets. Nature 464, 199-208 (2010)

2. Wen, X.-G. Choreographic entanglement dances: topological states of quantum matter. Science 363, 834 (2019).

3. Broholm, C. et al. Quantum spin liquid. Science 367, 263 (2020).

4. Takagi, H. et al. Concept and realization of Kitaev quantum spin liquids. Nat. Rev. Phys. 1, 246 (2019).

5. Tang, E., Mei, J.-W. \& Wen, X.-G. High-temperature fractional quantum Hall states. Phys. Rev. Lett. 106, 236802 (2011).

6. Bradlyn, B. et al. Topological quantum chemistry. Nature 547, 298 (2017).

7. Toth, S. et al. Electromagnon dispersion probed by inelastic $\mathrm{x}$-ray scattering in $\mathrm{LiCrO}_{2}$. Nat. Commun. 7, 13547 (2016).

8. Pouget, J. P. et al. Neutron-scattering investigations of the Kohn anomaly and of the phase and amplitude charge density wave excitations of the blue bronze $\mathrm{K}_{0.3} \mathrm{MoO}_{3}$. Phys. Rev. B 43, 8421 (1991).

9. Hoesch, M. et al. Giant Kohn anomaly and the phase transition in charge density wave $\mathrm{ZrTe}_{3}$. Phys. Rev. Lett. 102, 086402 (2009).

10. Miao, H. et al. Incommensurate phonon anomaly and the nature of charge density waves in cuprates. Phys. Rev. X 8, 011008 (2018).

11. Aynajian, P. et al. Energy gaps and Kohn anomalies in elemental superconductors. Science 319, 1509 (2008).

12. Mross, D. F. \& Senthil, T. Charge Friedel oscillations in a Mott insulator. Phys. Rev. B 84, 041102 (2011)

13. Grissonnanche, G. et al. Giant thermal Hall conductivity in the pseudogap phase of cuprate superconductors. Nature 376, 571 (2019).

14. Samajdar, R. et al. Enhanced thermal Hall effect in the square-lattice Néel state. Nat. Phys. 15, 1290 (2019).

15. Han, J. H., Park, J.-H. \& Lee, P. A. Consideration of thermal Hall effect in underdoped cuprates. Phys. Rev. B 99, 205157 (2019).

16. Kitaev, A. Anyons in an exactly solved model and beyond. Ann. Phys. 321, 2 (2006).

17. Sandilands, L. J., Tian, Y., Plumb, K. W., Kim, Y.-J. \& Burch, K. S. Scattering continuum and possible fractionalized excitations in $\alpha-\mathrm{RuCl}_{3}$. Phys. Rev. Lett. 114, 147201 (2015).

18. Nasu, J., Knolle, J., Kovrizhin, D. L., Motome, Y. \& Moessner, R. Fermionic response from fractionalization in an insulating two-dimensional magnet. Nat. Phys. 12, 912-915 (2016)

19. Banerjee, A. et al. Proximate Kitaev quantum spin liquid behaviour in a honeycomb magnet. Nat. Mat. 15, 733-740 (2016).

20. Hermanns, M. et al. Physics of the Kitaev model: fractionalization, dynamic correlations, and material connections. Annu. Rev. Condens. Matter Phys. 9, 17-33 (2017)

21. Banerjee, A. et al. Neutron scattering in the proximate quantum spin liquid $\alpha-$ $\mathrm{RuCl}_{3}$. Science 356, 1055-1059 (2017).

22. Do, S.-H. et al. Majorana fermions in the Kitaev quantum spin system $a$ $-\mathrm{RuCl}_{3}$. Nat. Phys. 13, 1079-1084 (2017)

23. Jansa, N. et al. Observation of two types of fractional excitation in the Kitaev honeycomb magnet. Nat. Phys. 14, 786 (2018).

24. Widmann, S. et al. Thermal dynamic evidence of fractionalized excitations in a-RuCl 3 . Phys. Rev. B 99, 094415 (2019).

25. Laurell, P. \& Okamoto, S. Dynamical and thermal magnetic properties of the Kitaev spin liquid candidate $\alpha-\mathrm{RuCl}_{3}$. npj Quantum. Materials 5, 2 (2020).

26. Ye, M., Halász, G. B., Savary, L. \& Balents, L. Quantization of the thermal Hall conductivity at small Hall angles. Phys. Rev. Lett. 121, 147201 (2018).

27. Vinkler-Aviv, Y. \& Rosch, A. Approximately quantized thermal Hall effect of chiral liquids coupled to phonons. Phys. Rev. X 8, 031032 (2018).

28. Metavitsiadis, A. \& Brenig, W. Phonon renormalization in the Kitaev quantum spin liquid. Phys. Rev. B 101, 035103 (2020).

29. Ye, M. et al. Phonon dynamics in the Kitaev spin liquid. Phys. Rev. Res. 2, 033180 (2020).

30. Kasahara, Y. et al. Majorana quantization and half-integer thermal quantum Hall effect in a Kitaev spin liquid. Nature 559, 227-231 (2018).

31. Yamashita, M., Kurita, N. \& Tanaka, H. Sample dependence of the half-integer quantized thermal Hall effect in the Kitaev candidate $\alpha-\mathrm{RuCl}_{3}$. Phys. Rev. B 102, 220404 (2020).

32. Nasu, J., Yoshitake, J. \& Motome, Y. Thermal transport in the Kitaev model. Phys. Rev. Lett. 119, 127204 (2017).

33. Jackeli, G. \& Khaliullin, G. Mott insulators in the strong spin-orbit coupling limit: from Heisenberg to a quantum compass and Kitaev models. Phys. Rev. Lett. 102, 017205 (2009).
34. Yoshitake, J. et al. Fractional spin fluctuations as a precursor of quantum spin liquids: Majorana dynamical meanfield study for the Kiteav model. Phys. Rev. Lett. 117, 157203 (2016).

35. Leahy, I. A. et al. Anomalous thermal conductivity and magnetic torque response in the honeycomb magnet $\alpha-\mathrm{RuCl}_{3}$. Phys. Rev. Lett. 118, 187203 (2017).

36. Hentrich, R. et al. Unusual phonon heat transport in $\alpha-\mathrm{RuCl}_{3}$ : strong spinphonon scattering and field-induced spin gap. Phys. Rev. Lett. 120, 117204 (2018).

37. Kasahara, Y. et al. Unusual thermal Hall effect in a Kitaev spin liquid candidate $\alpha-\mathrm{RuCl}_{3}$. Phys. Rev. Lett. 120, 217205 (2018).

38. Banerjee, A. et al. Excitations in the field-induced quantum spin liquid state of a-RuCl3. njp Quantum Mater. 3, 8 (2018).

39. Balz, C. et al. Finite field regime for a quantum spin liquid in $a-\mathrm{RuCl}_{3} . P h y$ s. Rev. B 100, 060405 (2019).

40. Reschke, $\mathrm{S}$. et al. Terahertz excitations in $\alpha-\mathrm{RuCl}_{3}$ : Majorana fermions and rigid-plane shear and compression modes. Phys. Rev. B 100, 100403(R) (2019).

41. Miao, H. et al. Observation of double Weyl phonons in parity breaking FeSi. Phys. Rev. Lett. 121, 035302 (2018).

42. Zhang, T. T. et al. Phononic helical nodal lines with $P T$ protection in $\mathrm{MoB}_{2}$. Phys. Rev. Lett. 123, 245302 (2019).

43. Yoshitake, J., Masu, J. \& Motome, Y. Fractional spin fluctuations as a precursor of quantum spin liquids: Majorana dynamical mean-field study for the Kitaev model. Phys. Rev. Lett. 117, 157203 (2016).

44. Rusnačko, J., Gotfryd, D. \& Chaloupka, J. Kitaev-like honeycomb magnets: global phase behavior and emergent effective models. Phys. Rev. B 99, 064425 (2019).

45. Hickey, C. \& Trebst, S. Emergence of a field-driven $U(1)$ spin liquid in the Kitaev honeycomb model. Nat. Commun. 10, 530 (2019).

46. Teng, Y. et al. Unquantized thermal Hall effect in quantum spin liquids with spinon Fermi surfaces. Phy. Rev. Research 2, 033283 (2020).

47. Winter, S. M. et al. Breakdown of magnons in a strongly spin-orbital coupled magnet. Nat. Commun. 8, 1152 (2017).

48. Kitagawa, K. et al. A spin-orbital-enhanced quantum liquid on a honeycomb lattice. Nature 554, 341 (2018).

49. Han, T.-H. et al. Fractionalized excitations in the spin-liquid state of a kagome-lattice antiferromagnet. Nature 492, 406 (2012).

50. Shen, Y. et al. Evidence for a spinon Fermi surface in a triangular-lattice quantum-spin-liquid candidate. Nature 540, 559 (2016).

51. Grissonnanche, G. et al. Chiral phonons in the pseudogap phase of cuprates Nat. Phys. 16, 1108 (2020).

52. May, A. F. et al. A practical guide for crystal growth of van der Waals layered materials. Journal of Applied Physics 128, 051101 (2020).

\section{Acknowledgements}

We thank T. Berlijn, H. Ding, J. K. Keum, G. Kotliar, S. Nagler, N. Perkins, and A. Tennan for stimulating discussions. This research at Oak Ridge National Laboratory (ORNL) was sponsored by the U.S. Department of Energy, Office of Science, Basic Energy Sciences, Materials Sciences and Engineering Division (IXS data analysis, material synthesis and data interpretation) and by the Laboratory Directed Research and Development Program of ORNL, managed by UT-Battelle, LLC, for the U.S. Department of Energy (IXS experiment). Part of IXS data interpretation work at Brookhaven National Laboratory was supported by the U.S. DOE, Office of Science, Office of Basic Energy Sciences, Materials Sciences, and Engineering Division under Contract No. DE-SC0012704. This research used resources of the Advanced Photon Source, a U.S. Department of Energy (DOE) Office of Science User Facility, operated for the DOE Office of Science by Argonne National Laboratory under Contract No. DE-AC02-06CH11357. Extraordinary facility operations were supported, in part, by the DOE Office of Science through the National Virtual Biotechnology Laboratory, a consortium of DOE national laboratories focused on the response to COVID-19, with funding provided by the Coronavirus CARES Act. T.T.Z. and S.M. acknowledge the supports from Tokodai Institute for Element Strategy (TIES) funded by MEXT Elements Strategy Initiative to Form Core Research Center. T.T.Z. also acknowledge the support by Japan Society for the Promotion of Science (JSPS), KAKENHI Grant No. 21K13865. S.M. also acknowledges support by JSPS KAKENHI Grant No. JP18H03678. G.B.H. and S.O. were supported by the U.S. Department of Energy, Office of Science, National Quantum Information Science Research Centers, Quantum Science Center. D.G.M. acknowledges support from the Gordon and Betty Moore Foundation's EPiQS Initiative, Grant GBMF9069.

\section{Author contributions}

H. M. conceived and designed the study. H.L., A.S., G.F., D.G.M., J.K.K., H.N.L., M.P.M.D., and H.M. performed the IXS experiment. H.L. and H.M. analyzed the IXS data. T.T.Z., S.M., G.B.H., and S.O. performed the DFT calculations and theoretical analysis. J.Q.Y. and D.M. synthesized the high-quality single crystal samples. H.L., T.T.Z., M.P.M.D., and H.M. prepared the manuscript with inputs from all authors. 


\section{Competing interests}

The authors declare no competing interests.

\section{Additional information}

Supplementary information The online version contains supplementary material available at https://doi.org/10.1038/s41467-021-23826-1.

Correspondence and requests for materials should be addressed to H.M.

Peer review information Nature Communications thanks the anonymous reviewers for their contribution to the peer review of this work.

Reprints and permission information is available at http://www.nature.com/reprints

Publisher's note Springer Nature remains neutral with regard to jurisdictional claims in published maps and institutional affiliations. (c) (i) Open Access This article is licensed under a Creative Commons Attribution 4.0 International License, which permits use, sharing, adaptation, distribution and reproduction in any medium or format, as long as you give appropriate credit to the original author(s) and the source, provide a link to the Creative Commons license, and indicate if changes were made. The images or other third party material in this article are included in the article's Creative Commons license, unless indicated otherwise in a credit line to the material. If material is not included in the article's Creative Commons license and your intended use is not permitted by statutory regulation or exceeds the permitted use, you will need to obtain permission directly from the copyright holder. To view a copy of this license, visit http://creativecommons.org/ licenses/by/4.0/.

(C) The Author(s) 2021 